\title{
Texture Synthesis using Energy Compaction Property of Different Transforms
}

\author{
D. Ramesh Varma \\ Assistant Professor, ECE \\ Shri Vishnu Engineering \\ College for Women
}

\author{
K. Pavan Raju \\ Assistant Professor, CSE \\ Shri Vishnu Engineering \\ College for Women
}

\author{
M. V. R. V Prasad \\ Assistant Professor, ECE \\ Vishnu Institute of Technology
}

\begin{abstract}
Image processing is one of the trending issues in the world of big data. The discovery of data from an image is a complex task in everyone's day to day life. In this paper, the texture synthesis is done with the help of energy compaction property of various transforms. The images are subjected to various combinations of transformations like DCT, DWT and Daubechies. The energy compaction of these transforms is explained in this paper. This property is used for restoring the images which are blurred due to atmospheric turbulence, motion blur and the images which are affected due to noise present in the channel. From the experiments, the DCT is having good energy compaction, but instead of using twodimensional transform, three-dimensional transform (2D + 1D) will give the better results when compared to the $2 \mathrm{D}$ transform for synthesizing the textures.
\end{abstract}

\section{Keywords}

Texture synthesis, Energy Compaction, Transform.

\section{INTRODUCTION}

Today, we are living in the era of big data. The discovery, interpretation and gaining knowledge from the data is a crucial task in everyone's day to day life. The texture synthesis which is nothing but restoring the image from its degraded version is done in different perspectives. The textures in an image play a crucial role in image restoration [1]. Generally, images are corrupted due to channel noise and blur effect while capturing the image. The restoration of image is done by synthesizing the textures with the help of energy compaction property of different transforms.

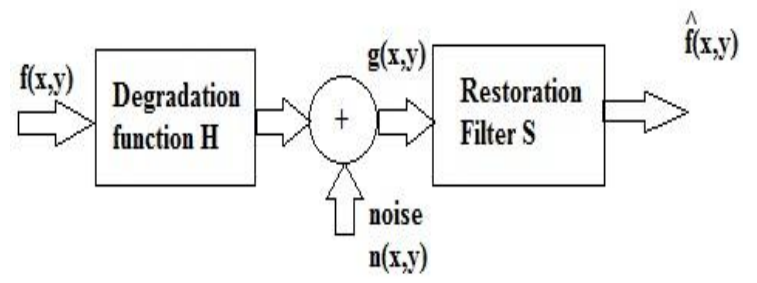

Fig.1.1. Restoration of Image

\section{IMAGE TRANSFORMS}

There are various types of image transforms. They are DFT, DCT, HAAR, DAUBECHIES, Bi-ORTHOGONAL etc..,

\subsection{DFT}

Now a day's images are represented in digital form, hence it is very urgent to have discrete form of Fourier transform [2]. This requirement is satisfied by the discrete Fourier transform. This transform takes data values which are regularly spaced as input and gives the value of the Fourier transform as output.
The 2D-DFT of a rectangular image $\mathrm{f}(\mathrm{x}, \mathrm{y})$ of size $\mathrm{X} \times \mathrm{Y}$ is represented as

$$
\mathrm{f}(\mathrm{x}, \mathrm{y}) \longrightarrow \mathrm{F}(\mathrm{p}, \mathrm{q})
$$

Energy compaction property of DFT is poor. The most of the energy is concentrated at the corners of transformed image [as shown in Fig.2.1] which is a complex task for synthesizing the textures of an image. Also, in DFT the signal is represented periodically. When coefficients are truncated, the signal will loose its form.

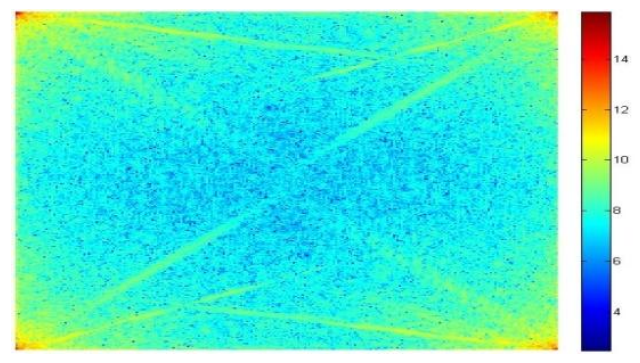

Fig.2.1. Energy Compaction of DFT

\subsection{DCT}

DCT is a part of real-valued discrete sinusoidal unitary transforms. It has a set of basis vectors which are sampled cosine functions. It is used for conversion of a signal into frequency components. It has many applications in image processing such as compression. The Discrete Cosine transform consists of good energy compaction for highly correlated data [as shown in Fig.2.2]. It puts more energy in few coefficients that is low frequency regions [3]. Based on the bandwidth requirements of receivers, DCT coefficients can be removed by the quantizer without the loss of quality of the image. Advantage of using DCT is that there are no discontinuities in it. In DCT, due to continuous periodic structure, the signal can withstand more coefficient truncation but still retain the desired shape.

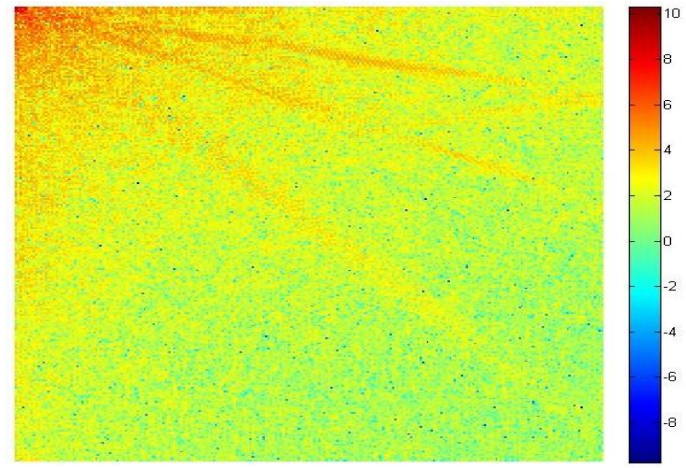

Fig.2.2. Energy Compaction of DCT 


\subsection{HAAR Transform}

The haar transform is given by using Haar functions. The basis elements of haar transform are $[-1,+1,0]$. There is no necessity of multiplications in the haar transform. It needs only additions. Many elements in the haar matrix are zeros. Therefore, the computational period is very short. Haar transform is faster than Walsh transform. Input and output lengths of haar transform should be of the same length. But, the length should be a power of 2. Haar transform is used to study the localized features of signals. The frequency components of input signal can be analyzed by using a property of the Haar function called orthogonal property. But the problem with the HAAR transform is that the energy compaction properties of the Haar transform are not very good [as shown in Fig.2.3] however, we will use haar transform as the vehicle which take us from the world of unitary transforms to that of multi-resolution analysis.
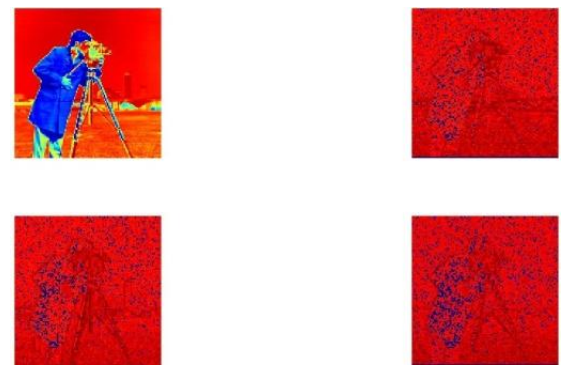

Fig.2.3. Energy Compaction of HAAR

\subsection{Daubechies Transform}

The Daubechies wavelets are the part of the family of orthogonal wavelets which defines a discrete wavelet transform (DWT) and it is given by a maximal number of dissipating points. With each type of wavelet of this class, there is a scaling function which produces an orthogonal multi-resolution analysis. The same with the case of daubechies transform as they perform poorly for textures. Energy compaction of daubechies is not good as shown in Fig.2.4.
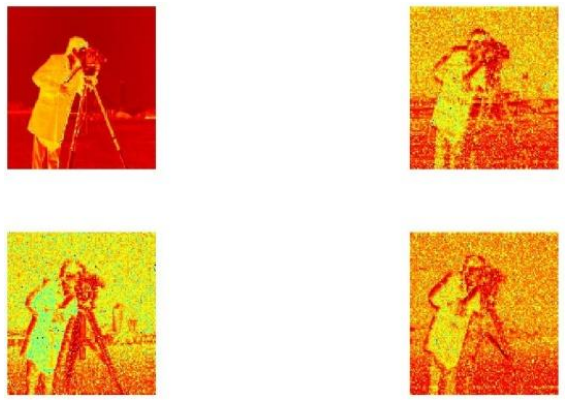

Fig.2.4. Energy Compaction of Daubechies (db8)
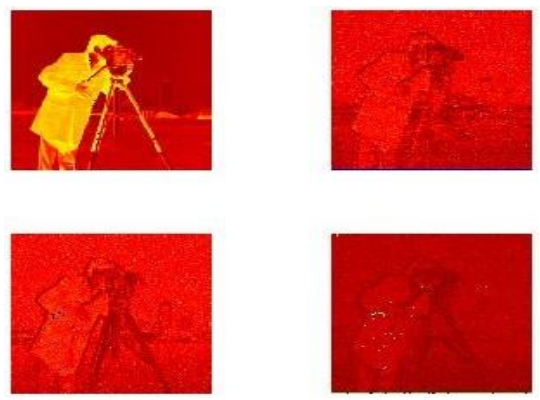

Fig.2.5. Energy Compaction of Daubechies (db2)

\section{ENERGY COMPACTION OF 3D IMAGE TRANSFORMS}

\subsection{D-DCT + 1D-HAAR}
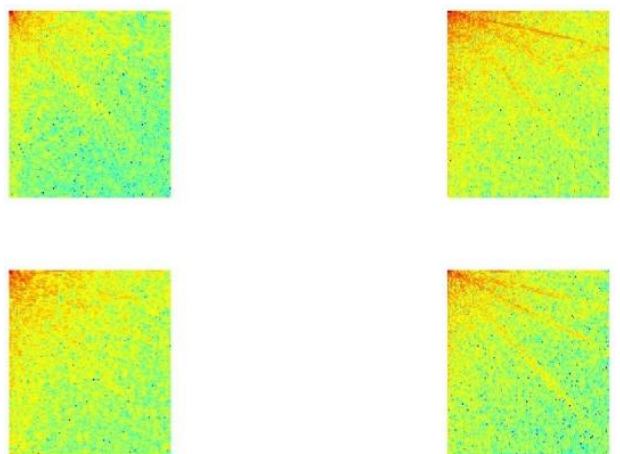

Fig.3.1. Energy Compaction of 2D-DCT + 1D-HAAR

From figure 3.1. it is clearly visible that the energy (Red colour region) is concentrated in the fewer coefficients. Because of this reason, it is able to synthesize the texture with fewer coefficients which reduces the complexity of restoring the image.

\subsection{D-DCT + 1D-daubechies (db8)}
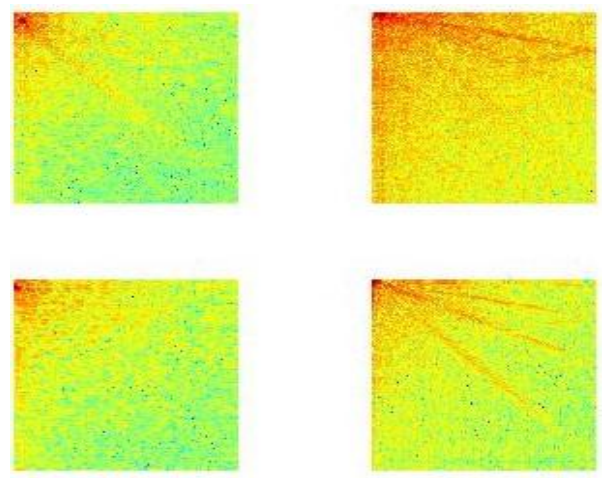

Fig.3.2. Energy Compaction of 2D-DCT + 1DDaubechies

From the above figure 3.2, it is clear that rather than using image transforms individually, it is better to use them in combinations.

\subsection{D-DCT + 1D-DFT}

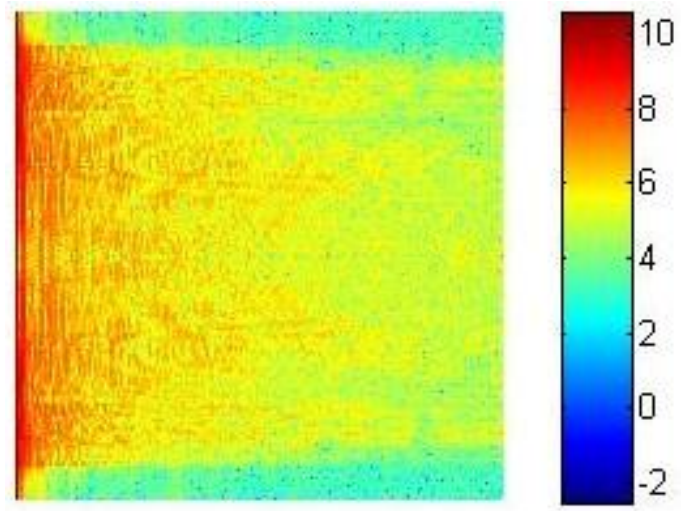

Fig.3.3. Energy Compaction of 2D-DCT + 1D-DFT 
The combination of the advantage of DCT and disadvantage of DFT gave mixed result [4]. It is somewhat better than performing individual DFT.

\subsection{D-DFT + 1D-HAAR}
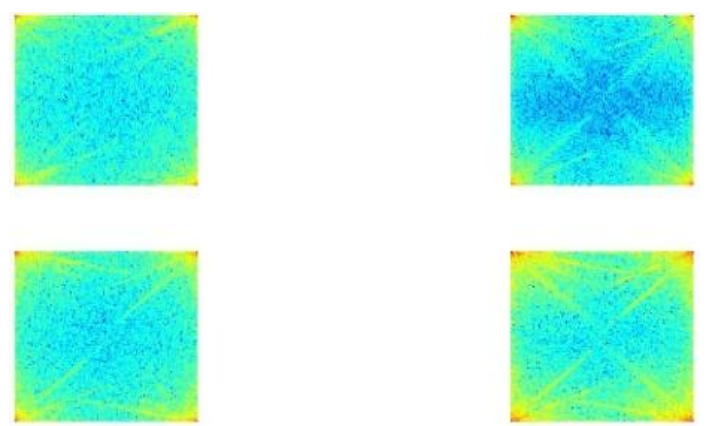

Fig.3.3. Energy Compaction of 2D-DFT + 1D-HAAR

\subsection{D-DFT + 1D-daubechies (db8)}
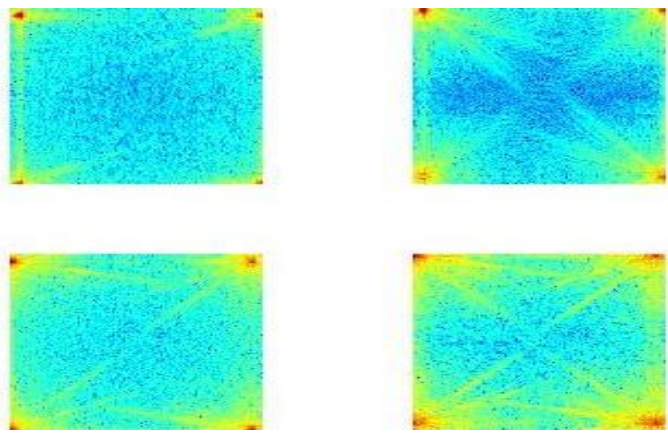

Fig.3.3. Energy Compaction of 2D-DFT + 1DDaubechies

From all the above figures, it is clear that discrete cosine transform is having higher energy compaction [Red colour region is concentrated in fewer coefficients] when compared to DFT, HAAR and Daubechies. So, 3D domain is established with the help of 2D-DCT + 1D other transforms. For synthesizing the textures, the data should be highly correlated.

\section{TEXTURE SYNTHESIS}

Synthesizing the textures is done in the perspective of restoring the image globally. This can be done in following steps.

Step1. Select the degraded image which is to be restored by synthesizing the texture regions.

Step2. Select the texture regions which are denoted by red colour window.

Step3. Select another region which is denoted by blue colour window around texture region for finding similar images

Step4. Divide the blue colour window into ' $n$ ' number of blocks

Step5. Calculate Euclidean distance between texture region and each block of region around texture region. The blocks which are having less Euclidean distance is taken as self-similar images.

Step6. Apply 2D-DCT $+1 \mathrm{D}$-other transforms (2D-DCT + 1D-HAAR give best result). Now, transform coefficients are obtained.
Step7. Apply inverse transform by which block-wise estimates are obtained for the each block of $n$ blocks. By averaging these block-wise estimates, a final estimate is obtained. For this to happen, energy compaction property plays a crucial role in synthesizing the textures.

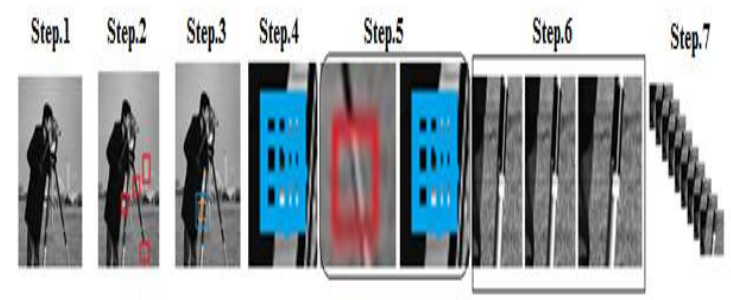

Fig.4.1. Texture synthesis using transforms

\section{PERFORMANCE METRICS}

In this paper, the image inverse problem image denoising task is performed [5-8]. The noise is removed from the image with the help of transforms. The restored image is visualized perfectly by synthesizing the textures.

\begin{tabular}{|c|c|c|}
\hline \multicolumn{3}{|c|}{ Table.1. Performance Metrics } \\
\hline IMAGE & $\begin{array}{c}\text { 3D } \\
\text { TRANSFOR } \\
\text { M }\end{array}$ & $\begin{array}{c}\text { PSNR } \\
\text { VALU } \\
\text { E }\end{array}$ \\
\hline \multicolumn{2}{|r|}{ 3D-DCT } & 32.82 \\
\hline & $\begin{array}{c}\text { 2D-DCT + 1D- } \\
\text { HAAR }\end{array}$ & 32.55 \\
\hline & $\begin{array}{l}\text { 2D-DCT + 1D- } \\
\text { Daubechies }\end{array}$ & 30.25 \\
\hline & $\begin{array}{c}2 \mathrm{D}-\mathrm{DCT}+1 \mathrm{D}- \\
\text { Bior } 1.5\end{array}$ & 30.01 \\
\hline & $\begin{array}{c}2 \mathrm{D}-\mathrm{DFT}+1 \mathrm{D}- \\
\mathrm{DCT}\end{array}$ & 29.26 \\
\hline \multirow[t]{2}{*}{ Cameraman } & $\begin{array}{c}\text { 2D-DFT + 1D- } \\
\text { HAAR }\end{array}$ & 29.14 \\
\hline & $\begin{array}{l}\text { 2D-DFT + 1D- } \\
\text { Daubechies }\end{array}$ & 27.29 \\
\hline
\end{tabular}




\begin{tabular}{|c|c|c|}
\hline \multicolumn{3}{|c|}{ Table.2. Performance Metrics } \\
\hline \multirow[t]{6}{*}{ IMAGE } & $\begin{array}{c}\text { 3D } \\
\text { TRANSFORM }\end{array}$ & PSNR VALUE \\
\hline & 3D-DCT & 31.52 \\
\hline & $\begin{array}{c}\text { 2D-DCT + 1D- } \\
\text { HAAR }\end{array}$ & 31.25 \\
\hline & $\begin{array}{l}\text { 2D-DCT + 1D- } \\
\text { Daubechies }\end{array}$ & 29.95 \\
\hline & $\begin{array}{l}\text { 2D-DCT + 1D- } \\
\quad \text { Bior } 1.5\end{array}$ & 29.31 \\
\hline & $\begin{array}{c}\text { 2D-DFT + 1D- } \\
\text { DCT }\end{array}$ & 28.66 \\
\hline \multirow[t]{2}{*}{ parrot } & $\begin{array}{c}\text { 2D-DFT + 1D- } \\
\text { HAAR }\end{array}$ & 28.54 \\
\hline & $\begin{array}{l}\text { 2D-DFT + 1D- } \\
\text { Daubechies }\end{array}$ & 26.29 \\
\hline
\end{tabular}

\section{RESULTS AND CONCLUSION}

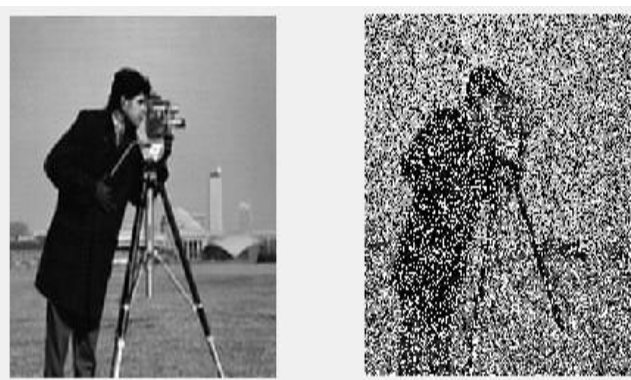

Fig.6.1. Image denoising using 3D-DCT
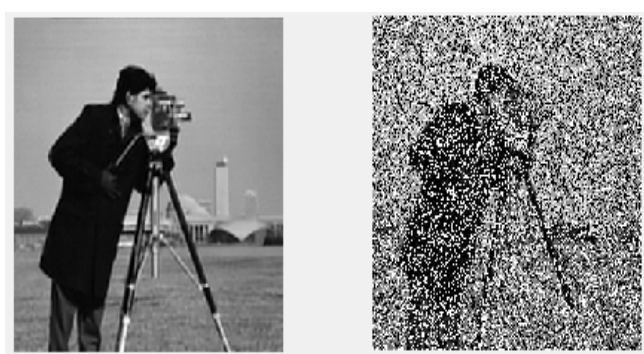

Fig.6.2. Image denoising using 2D-DCT + 1D-HAAR
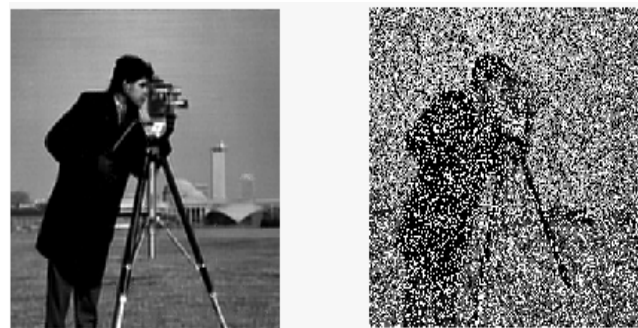

Fig.6.3. Image denoising using 2D-DCT + 1DDaubechies

In this paper, energy compaction property of various transforms is analysed for synthesizing the textures which is nothing but restoration of global details of an image. From the discussions a conclusion is made that the discrete cosine transform gave the better peak signal to noise ratio and best visualization when compared to other transforms. These analyses can be used for future of several image inverse problems like Deblurring the image and image interpolation.

\section{ACKNOWLEDGEMENT}

We feel quiet delighted to have great opportunity to thank authors for providing necessary information which leads to successful completion of our work. We would also thank our principal, Head of the departments for providing necessary facilities at the college. We would also thank our professors, friends and family for being so supportive throughout the project.

\section{REFERENCES}

[1] M.R. Banham and A.K. Katsaggelos,"Digital Image Restoration," IEEE Transactions, signal processing vol.14,no.2, pp.24-41, Mar 1997

[2] "Image Transforms" Digital Image processing, E.Essakirajan, S.Jayaraman.

[3] Image Restoration using 3-Dimensional Discrete Cosine Transform, International Journal of Computer Applications (0975 - 8887) Volume 156 - No 9, December 2016.

[4] A New Weighted Average Filter for removing camera shake, International Journal of Computer Applications, vol 156-No.9, December 2016.

[5] K. Dabov, A. Foi, V. Katkovnik, and K. Egiazarian, "Image denoising by sparse 3D transform-domain collaborative filtering," IEEE Transactions for Image Processing, Aug 2007.

[6] A.Buades, B.coll and J.M. Morel, "A non-local algorithm for image denoising"

[7] A. Foi, V. Katkovnik, and K. Egiazarian, "Pointwise shape-adaptive DCT for high-quality denoising and deblocking of grayscale and color images,"

[8] M.Mohan Babu, M.N Giri Prasad, M.V Subramanyam "A New Approach for SAR Image denoising", International Journal of Electrical and Computer Engineering (IJECE) Vol 5, No 5, 2015. 